\title{
Vortices capturing matter: a classroom demonstration
}

\author{
T Téli ${ }^{1,2,3}$, M Vincze $^{1,2}$ and I M Jánosi ${ }^{1,4}$ \\ 1 von Kármán Laboratory, Eötvös University, 1117, Pázmány P. s. 1/A, Budapest, Hungary \\ 2 MTA-ELTE Theoretical Physics Research Group, 1117, Pázmány P. s. 1/A, Budapest, \\ Hungary \\ ${ }^{3}$ MTA-ELTE Physics Education Research Group, 1117, Pázmány P. s. 1/A, Budapest, \\ Hungary \\ ${ }^{4}$ Department of Physics of Complex Systems, Eötvös University, 1117, Pázmány P. s. 1/A, \\ Budapest, Hungary \\ E-mail: tel@general.elte.hu
}

\begin{abstract}
When injecting dye into a vortex, one finds that the dye remains captured around the vortex core over minutes, despite the fact that the vortex (and the dyed region) is strongly time-dependent. According to a recent theory, three-dimensional time-dependent vortices should be defined as rotating, material-holding regions of the fluid. Vortices generated by commercial magnetic stirrers appear to demonstrate this material-holding property. These experiments can be carried out with tap water and food dye in any school. They help make students familiar with the material holding character of vortices. This is a recently understood, rather robust, property, observable also in vortices occurring in nature.
\end{abstract}

\section{The phenomenon}

Vortices generated by some kind of stirrer device are standard tools for illustrating the structure of tornadoes in schools (see, e.g.: [1]). In the von Kármán Laboratory for Environmental Flows at Eötvös University [2] for this purpose we use a vortex generated by a magnetic stirrer. This experiment is always a hit among all types of audiences, ranging from kindergarten kids, via high school and university students to parents.

Commercial magnetic stirrers are commonly used to mix different materials dissolved in fluids. The rotation of the magnet brings a magnetic stirrer bar, whose rotational axis is not fixed, into rotation at the bottom of a container. If the container is filled with a liquid, the bar generates fluid motion, which is believed to cause efficient stirring and mixing. A striking pattern of such flows is a big vortex accompanied with a depression of the free surface, the funnel. With our experiments we demonstrate that there is a strong downwelling in the middle, underneath the funnel and that particles lighter than the ambient fluid are attracted towards the vortex axis.

The most surprising effect appears, however, when one drops dye into the central region of the vortex from the surface, marked by the location of the funnel: the injected material stretches in the vertical direction in a compactly arranged manner, forming a cylinder of high dye concentration lasting over minutes (figure 1(a)). All this happens despite the fact that the vortex (and the dyed domain) is time-dependent since the stirrer bar is not fixed to an axis. If a certain amount of dye happens to enter a region outside a critical radius measured from the center, it becomes rapidly 


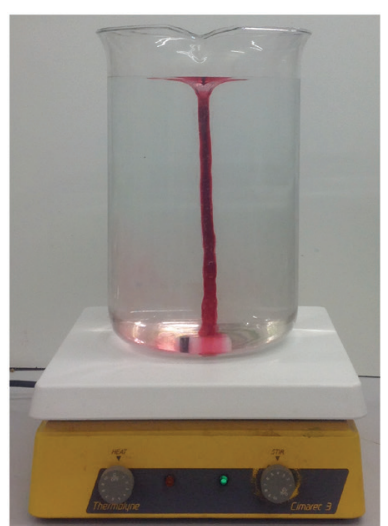

a)

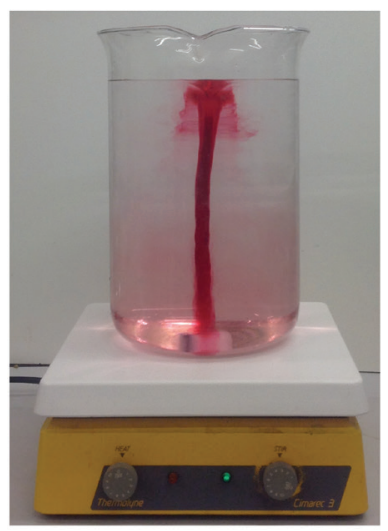

b)

Figure 1. Dye entering a central region marked by the surface depression (a) remains in a cylindrical shaped region over surprisingly long times (minutes). When injecting dye somewhat off the vortex center close to the surface (b), a transient pattern, a collar develops, but dye entering further away rapidly disperses. The white rod at the bottom with a black stripe on it is the stirrer bar.

diluted, and hardly visible. When dye happens to come close to the center on the surface, but is outside a certain critical radius, it remains localised but does not stretch downward along a cylindrical surface and becomes washed away sooner or later. We call this transient pattern a collar (figure 1(b)).

Up to 2017, we had to conclude our shows by saying that we were not aware of any clear explanation of the dye-capturing phenomenon.

\section{A new view on vortices}

It has long been known that the traditional definition of vortices, or of their size, is not unambiguous $[3,4]$. The definitions are typically based on the concept of vorticity, an instantaneous property of the flow. A recent development in fluid dynamics focuses on dye patterns, i.e. on properties related to the temporal history of the fluid motion, so-called Lagrangian Coherent Structures [5]. As a spinoff of this cutting-edge development, a new, objective definition of three-dimensional timedependent vortices appeared in the literature [6]. The content of this paper was, from the point of view of our vortex-related laboratory experience, a kind of relevation. The main statements of this new theory can be translated in the language of experimental physics as follows. Time-dependent vortices (like ours) possess a basic property, even more relevant than just the rotation of the fluid, namely they keep the material (water or dyed water in our case) in their center. The authors of [6] suggest that

- there is a set of concentric cylindrical-like surfaces inside the vortex (all with material holding property), and

- there exists, however, an outermost smooth surface with this property, and they claim that this is the edge of the vortex (figure 2).

The diameter of this outermost smooth surface sets the size of the vortex, but its precise value is not predicted by the theory.

Our traditional experiments provide thus evidence supporting this new theory in a very elementary set-up. By a set of many repeated injections we experimentally determined in [7] the diameter $d$ of the vortex edge, within which dye remains captured. When searching for another quantity to which the vortex diameter can be related, we found that this size is practically the same as the diameter $b$ of the funnel appearing on the free surface.

\section{A qualitative explanation}

For a deeper understanding, let us consider the funnel-shaped depression of the free water surface, associated with a vortex (figure 3 ). This funnel's characteristic diameter $b$ can then be defined as its width at its half depth. In the vicinity of the minimum of the surface displacement the shape is parabolic. A rotating water body with free surface is known to exhibit a surface deformation that is paraboloidal in shape. Thus, observing the shape of the moving funnel on the surface at a given instant, one can find a region in which the 
Vortices capturing matter: a classroom demonstration

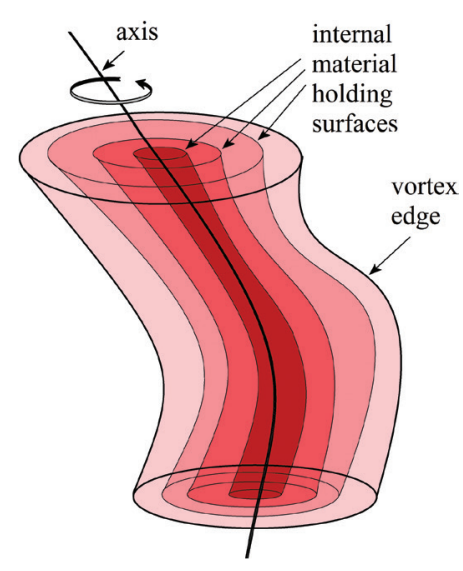

Figure 2. Schematic diagram illustrating the main components of a moving vortex: its axis, the material holding cylindrical-like surfaces in the inside, and the outermost smooth material holding surface, the vortex edge (after [6])

flow can be considered to rotate with constant angular velocity (be in so-called solid body rotation). Here the cross section of the surface shape (green line in figure 3 ) is approximately parabolic (dashed curve). The boundaries of this domain are denoted with vertical orange lines. In this region fluid particles rotate at a constant angular velocity relative to the center. Their path in the horizontal plane is circular, fluid particles from neighboring circles do not mix. Each cylindrical tube extending from such a circle downward is obviously a material holding surface. Further out, the angular velocity is no longer constant, but the motion is still coherent in the sense that fluid particles move along closed orbits (the flow is laminar). For moving vortices this zone has an outer boundary, namely the vortex edge of diameter $d$ introduced in the previous chapter (figure 2). Further out coherence breaks down, orbits become complicated and irregular; the vortex edge is thus the boundary between coherent and incoherent fluid motion. As our experiments show, this diameter is approximately equal to the width $b$ of the funnel: $d=b$, and the transition to incoherent flow is indicated by our observation concerning the collar (figure 1(b)).

\section{Testing stability}

The dye-holding property is surprisingly robust, we provide here suggestions for experiments to demonstrate this. When the stirrer bar is not in the

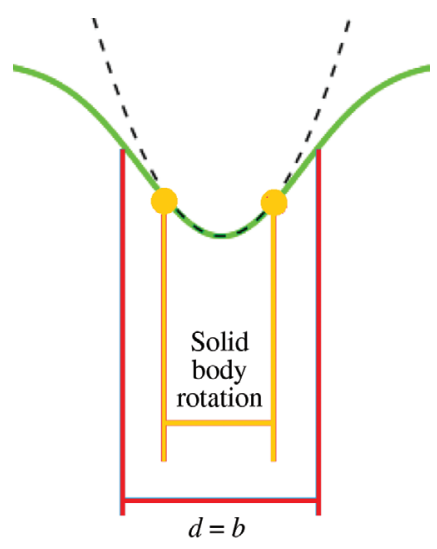

Figure 3. Schematic diagram of the instantaneous shape of the funnel on the surface. In the range marked with vertical orange lines the surface depression associated with a vortex is well approximated by a parabola (dashed line). Within this range the fluid is in solid body rotation. The domain of coherent (laminar) fluid motion extends somewhat further, to the diameter $d$ of the outermost material holding cylinder, the edge of the vortex (well approximated by the diameter $b$ of the funnel on the surface). Outside of this, coherent rotation is destroyed.

middle of the cylindrical container, injected dye traces out a tilted vortex which captures dye for a long time (figure 4(a)).

One can prepare a cylindrically symmetric dye column, as described initially, and then shift the container suddenly or tilt it for a short time. These movements result in a snake vortex (figure 4(b), the dyed water column start carrying out a wavy motion. By immersing a rod into the fluid and approaching it towards a vertical dye cylinder, one observes that even before the rod touches the outermost closed dye surface, it becomes destructed and an originally inner surface becomes the new outermost one. A complete destruction takes place when the pattern is cut through with the rod (figure 4(c)). Surprisingly enough, however, after the removal of the rod, the original cylinder is recovered, even if in a much paler form since most of the dye escaped the central region due to the downward flow. The water-holding property is thus robust and recovers itself once the original flow is re-established. After seeing this behavior of the vortex it is somewhat comical to think about the original purpose of the magnetic stirrer used in the experiment, that is to mix materials uniformly within 


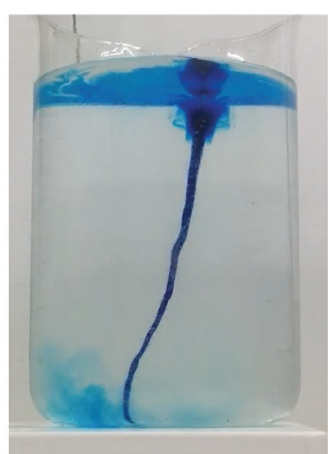

(a)

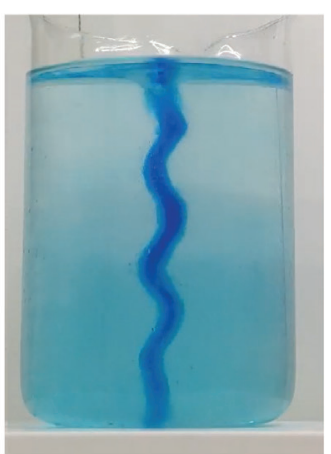

(b)

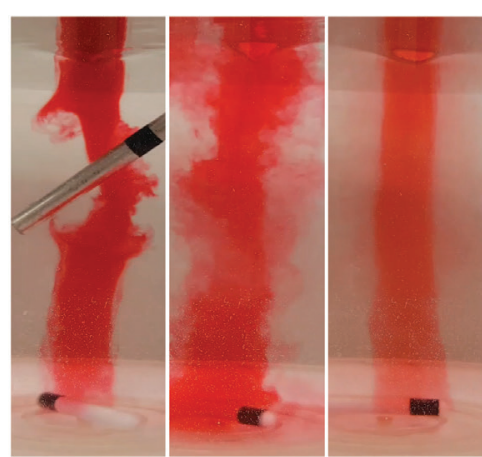

(c)

Figure 4. A vortex captures dye even if it is tilted (a), if it becomes a snake vortex after 'kicking' the container, or if it is cut through by a rod (c).
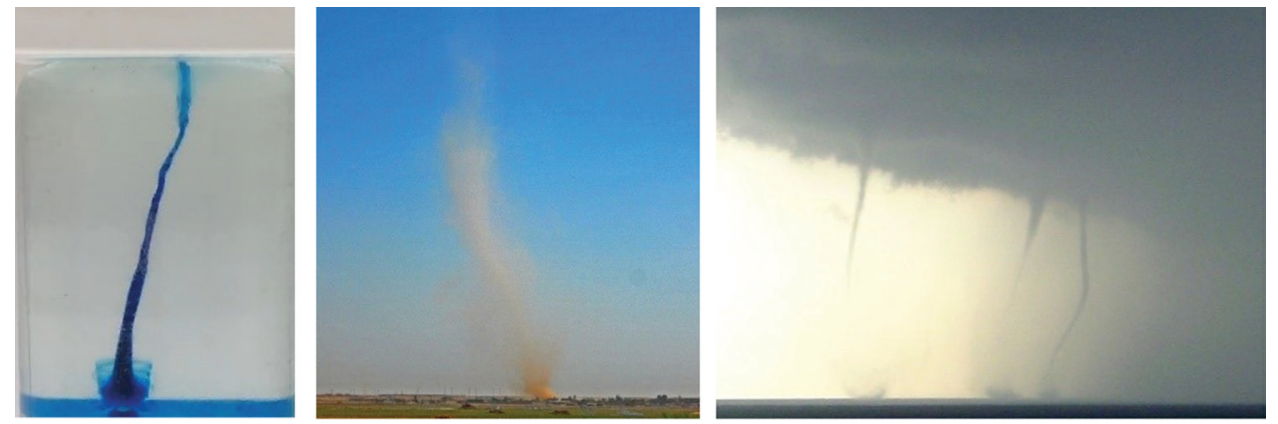

Figure 5. An experimental dye cylinder flipped upside down (left) strikingly resembles the pattern of dust devil [8] and water spouts [9], since in these vortices of nature suction occurs on the surface. (b) This Iraqi Dust Devil. jpg has been obtained by the author(s) from the Wikipedia website, where it is stated to have been released into the public domain. It is included within this article on that basis. (c) This Three waterspouts Kijkduin.jpg has been obtained by the author(s) from the Wikipedia website, where it is stated to have been released into the public domain. It is included within this article on that basis.

the cylindrical tank; apparently, this is just the opposite of what is happening close to the axis.

\section{Comparing with vortices in nature}

Vortices generated by our experiment strongly resemble those occurring in nature. Consider for example dust devils and water spouts. The dye in these cases corresponds to the material the vortex is named after: sand and water, respectively, more precisely a strongly air diluted mixture of these materials. When comparing a photo of a dye column taken from our experiments with those available on the internet for dust devils and water spouts (see e.g. [8,9], respectively) the similarity is clear but one component does not fit. In our examples the collar is on the top of the vortex

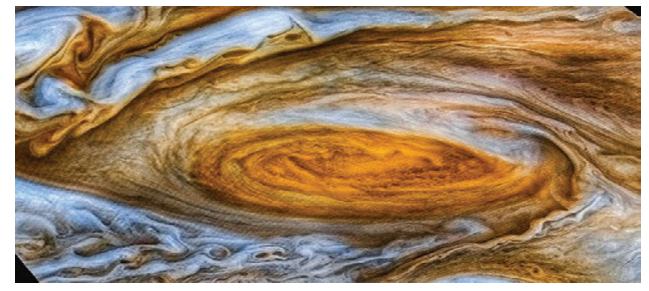

Figure 6. The Great Red Spot [13] is a gigantic (roughly Earth-sized) vortex in the atmosphere of Jupiter whose constant color is an indication of its matter-capturing (black hole like) property. Reproduced from [13]. CC BY 2.0.

while in the two examples taken from nature it is on the bottom. When recalling the appearance of a collar (see figure 1(b)), we realize that it is formed by dye injected outside of the vortex 


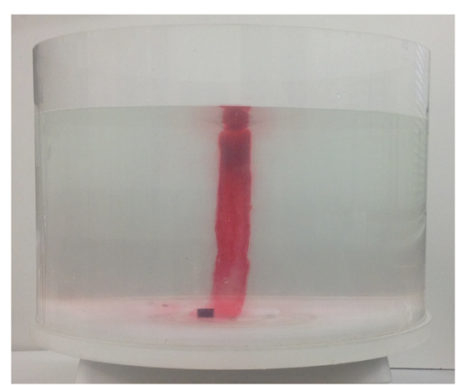

(a)

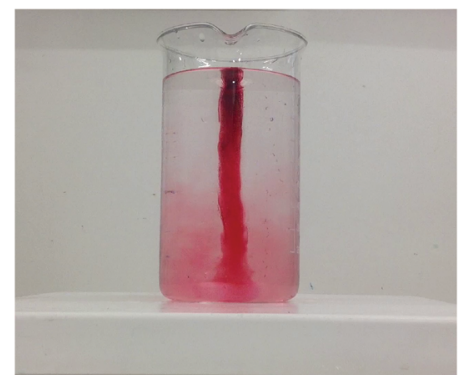

(c)

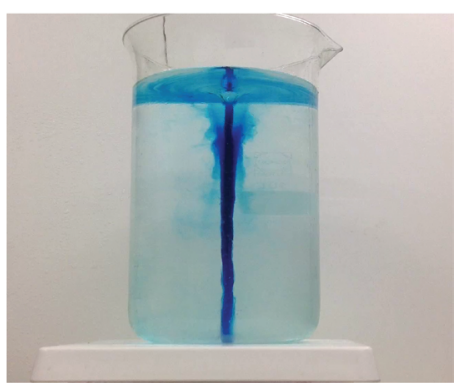

(b)

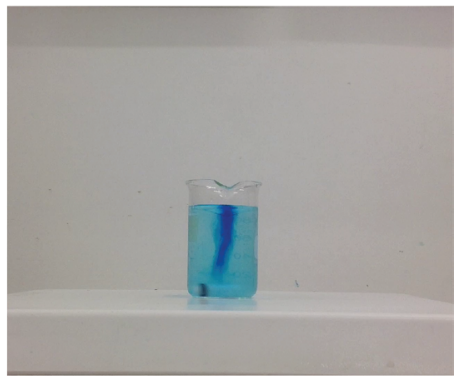

(d)

Figure 7. The material holding property of vortices can be demonstrated practically in any cylindrical container. The radius $(R)$ and height $(H)$ of the containers shown in panels (a)-(d) is in $\mathrm{cm} R, H=22,24 ; 12,16 ; 5,8 ; 2,2.7$. The length of the corresponding stirrer bars is $8,5,4,3 \mathrm{~cm}$, the frequencies and the lifetimes of a small amount of dye range from 7 to $4 \mathrm{~Hz}$ and 4 to $0.5 \mathrm{~min}$, respectively.

core, wherefrom dye is transported away outside of the vortex and becomes thus diluted. In our magnetic stirrer generated vortices fluid is moving downwards, but both sand and water is sucked upwards. A full analogy between these vortices and our laboratory vortices arises thus if we consider a 180-degree-rotated photograph of the latter (figure 5(a)).

The case of tornados is different since in their middle air is moving downwards, and the collar sometime visible due to water vapor (see e.g. [10]) appears just below the cloud bottom. These vortices correspond therefore to our laboratory vortices without any rotation.

\section{6. 'Black hole' property of vortices}

Research on lagrangian coherent structures lead to the conclusion that there is a mathematically founded similarity between the edge of twodimensional vortices and the edge of cosmological black holes [11]. To illustrate this analogy, class-room demonstrations were suggested in [12]. Using vortices similar to those we are using, the authors pointed out that shadows formed by illuminating these vortices are analogous to the gravitational action of black holes on light and surrounding matter. After the publication of [6] we can say that three-dimensional vortices also exhibit black hole like properties: they capture matter, as illustrated by all our examples. In fact, this feature is not restricted to vortices stretching between the surface and the bottom. Notable examples are oceanic eddies [6] detached e.g. from the Gulf Stream which are known to conserve their degree of salinity over years. or the Great Red Spot of Jupiter [13] (figure 6), a vortex keeping its red color over centuries, just to name a few.

\section{Summary}

Vortices are the building blocks of fluid flows, understanding their properties is relevant both for our everyday life and for our global environment. It is surprising that a basic, intuitively plausible property was scientifically established only a few years ago. It is fortunate that this material holding property is eye catching, and is easy to demonstrate in classrooms. To illustrate this, we present figure 7 to show that the phenomenon can be found in containers of any size realistic to find in schools. 
All in all, such inspiring experiments are highly recommendable for all schools, and we are in a lucky situation in which a recent scientific achievement can be converted into high school teaching, as a kind of educational reconstruction [14] of vortex theory.

\section{Acknowledgments}

The authors are grateful for Lyes Kadi for his useful contributions in the earlier stages of our vortex experiment project. We are grateful to Fred Feudel for suggesting to bring the container in motion in order to test the stability of vortices. Insightful discussions on Lagrangian Coherent Vortices with F Beron-Vera, M Farazmand and in particular with $G$ Haller are acknowledged. The project is funded by the Content Pedagogy Research Program of the Hungarian Academy of Sciences and by the NKFIH Office under Grants K-125171 and FK-125024. There are no conflicts of interest involved in this work. Certain images in this publication have been obtained by the author(s) from the Wikipedia/Wikimedia website, where they were made available under a Creative Commons licence or stated to be in the public domain. Please see individual figure captions in this publication for details. To the extent that the law allows, IOP Publishing [and full partner name (if applicable)] disclaim any liability that any person may suffer as a result of accessing, using or forwarding the image(s). Any reuse rights should be checked and permission should be sought if necessary from Wikipedia/Wikimedia and/or the copyright owner (as appropriate) before using or forwarding the image(s).

Received 5 August 2019, in final form 12 September 2019 Accepted for publication 11 October 2019 https://doi.org/10.1088/1361-6552/ab4d25

\section{References}

[1] www.fishpond.co.nz/Toys/Create-Your-OwnTornado-Experiment-Science-ExperimentSet-Number-One-Educational-Easter-GiftPresent-Science-Fun-Games-Toys-IdeaAge-8-Girls-Boys-Kids-Children-KidsLabs/9999336372519

[2] www.karman.elte.hu/index_eng.php

[3] Lugt H J 1983 Vortex Flow in Nature and Technology (Hoboken, NJ: Wiley)

[4] Guyon E, Hulin J P, Petit L and Mitescu C D 2015 Physical Hydrodynamics (Oxford: Oxford University Press)
[5] Haller G 2015 Lagrangian coherent structures Annu. Rev. Fluid Mech. 47 137-62

[6] Haller G, Hadjighasem A, Farazmand M and Huhn F 2016 Defining coherent vortices objectively from the vorticity J. Fluid Mech. 795 136-73

[7] Tél T, Kadi L, Jánosi I M and Vincze M 2018 Experimental demonstration of the waterholding property of three-dimensional vortices Eur. Phys. Lett. 12344001

[8] https://en.wikipedia.org/wiki/Dust_devil\#/ media/File:Iraqi_Dust_Devil.jpgSpouts

[9] https://en.wikipedia.org/wiki/Waterspout\#/ media/File:Three_waterspouts_Kijkduin.jpg

[10] https://regencyitc.co.uk/wp-content/ uploads/2013/01/Tornado-and-Lightning.jpg

[11] Haller G and Beron-Vera F J 2013 Coherent Lagrangian vortices: the black holes of turbulence J. Fluid Mech. 731 R4

[12] Barr I A, Bull A, O’Brien E, DrillsmaMilgrom K A and Milgrom L R 2016 Illuminating black holes Phys. Educ. 51043001

[13] www.flickr.com/photos/kevinmgill/28062689029

[14] Duit R, Gropengießer H, Kattmann U, Komorek M and Parchmann I 2012 The model of educational reconstruction - a framework for improving teaching and learning science Science Education Research and Practice in Europe ed D Jorde and J Dilon (Rotterdam: Sense Publishers) pp 13-37

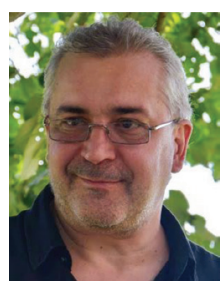

Imre M Jánosi was one of the co-founders of the von Karman Laboratory for Environmental Flows, in 1998. His research area is experimental fluid dynamics, and modelling of complex systems with many degrees of freedom. He often applies the tools of nonlinear time series analysis to analyse data from field campaigns or large data sets related to environmental and climate-related problems.

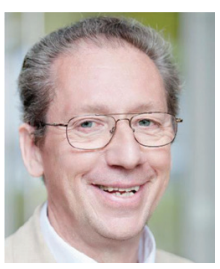

Tamas Tél was one of the co-founders of the von Karman Laboratory for Environmental Flows, in 1998. He contributed to nonlinear dynamical systems (chaos) theory; later his research interest turned to applications such as geophysical fluid flows, the dispersion of pollutants, or statistical climate dynamics. He also takes part in several experiments carried out in the von Karman Laboratory.

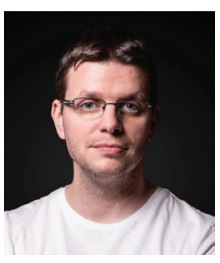

Miklós Vincze is a research fellow practically working in the von Karman Laboratory for Environmental Flows. His research mainly focuses on the experimental modelling of climaterelated geophysical flow problems, particularly buoyancy-driven convective and wave phenomena in rotating and stratified media in the form of tabletop-size laboratory set-ups. 